\title{
Preoperative physiotherapy for the prevention of respiratory complications after upper abdominal surgery: pragmatic, double blinded, multicentre randomised controlled trial
}

\author{
Ianthe Boden, 1,2 Elizabeth H Skinner, ${ }^{2,3}$ Laura Browning, ${ }^{2,3}$ Julie Reeve, ${ }^{4,5}$ Lesley Anderson, \\ Cat Hill, ${ }^{6}$ Iain K Robertson, ${ }^{7,8}$ David Story, ${ }^{9}$ Linda Denehy ${ }^{10,11}$
}

For numbered affiliations see end of article.

Correspondence to: I Boden ianthe.boden@ths.tas.gov.au Additional material is published online only. To view please visit the journal online.

Cite this as: BMJ 2018;360:j5916 http://dx.doi.org/10.1136/bmj.j5916

Accepted: 12 December 2017

\section{ABSTRACT}

OBJECTIVE

To assess the efficacy of a single preoperative physiotherapy session to reduce postoperative pulmonary complications (PPCs) after upper abdominal surgery.

\section{DESIGN}

Prospective, pragmatic, multicentre, patient and assessor blinded, parallel group, randomised placebo controlled superiority trial.

SETTING

Multidisciplinary preadmission clinics at three tertiary public hospitals in Australia and New Zealand.

PARTICIPANTS

441 adults aged 18 years or older who were within six weeks of elective major open upper abdominal surgery were randomly assigned through concealed allocation to receive either an information booklet ( $n=219$; control) or preoperative physiotherapy $(n=222$; intervention) and followed for 12 months. 432 completed the trial.

\section{INTERVENTIONS}

Preoperatively, participants received an information booklet (control) or an additional 30 minute physiotherapy education and breathing exercise training session (intervention). Education focused on PPCs and their prevention through early ambulation and self directed breathing exercises to be initiated immediately on regaining consciousness after surgery. Postoperatively, all participants received standardised early ambulation, and no additional respiratory physiotherapy was provided.

\section{WHAT IS ALREADY KNOWN ON THIS TOPIC}

Pulmonary complications are among the most serious negative outcomes after upper abdominal surgery and are associated with high mortality and costs

Trials have indicated that these complications might be prevented by preoperative physiotherapy education and breathing exercise instructions alone This evidence is limited by methodological weaknesses and poor generalisability within the context of modern advances in perioperative surgical practice

\section{WHAT THIS STUDY ADDS}

This trial provides strong evidence that a single preoperative physiotherapy session that educates patients on the reason and necessity to do breathing exercises immediately after surgery halves the incidence of postoperative respiratory complications

The number needed to treat to avoid postoperative pulmonary complications, including hospital acquired pneumonia, is 7 (95\% confidence interval 5 to 14 )

\section{MAIN OUTCOME MEASURES}

The primary outcome was a PPC within 14 postoperative hospital days assessed daily using the Melbourne group score. Secondary outcomes were hospital acquired pneumonia, length of hospital stay, utilisation of intensive care unit services, and hospita costs. Patient reported health related quality of life, physical function, and post-discharge complications were measured at six weeks, and all cause mortality was measured to 12 months.

\section{RESULTS}

The incidence of PPCs within 14 postoperative hospital days, including hospital acquired pneumonia, was halved (adjusted hazard ratio 0.48, $95 \%$ confidence interval 0.30 to $0.75, \mathrm{P}=0.001$ ) in the intervention group compared with the control group, with an absolute risk reduction of $15 \%$ (95\% confidence interval $7 \%$ to $22 \%$ ) and a number needed to treat of 7 (95\% confidence interval 5 to 14 ). No significant differences in other secondary outcomes were detected.

\section{CONCLUSION}

In a general population of patients listed for elective upper abdominal surgery, a 30 minute preoperative physiotherapy session provided within existing hospital multidisciplinary preadmission clinics halves the incidence of PPCs and specifically hospital acquired pneumonia. Further research is required to investigate benefits to mortality and length of stay.

TRIAL REGISTRATION

Australian New Zealand Clinical Trials Registry ANZCTR 12613000664741.

\section{Introduction}

Upper abdominal surgery is the most frequent major surgical procedure performed in developed countries. ${ }^{1}$ A postoperative pulmonary complication (PPC) is the most common serious complication after this type of surgery. ${ }^{2}$ The reported incidence is between $10 \%$ and $50 \%$ of patients. $^{2-12}$ The variability in reported PPC rates after upper abdominal surgery can be explained by the differing patient risk profiles studied and PPC definitions utilised. A PPC is strongly associated with increased mortality, morbidity, and healthcare costs. ${ }^{3-6}$ Pulmonary complications (including pneumonia and severe atelectasis) are caused by postoperative pathophysiological reductions in lung volumes, respiratory muscle function, mucociliary clearance, and pain inhibition of respiratory muscles. ${ }^{13}$ Breathing exercises may prevent PPCs by reversing these problems, although evidence is inconclusive. ${ }^{14}$ Findings may be limited by confounding combinations 
of both preoperative and postoperative interventions. Timing may be a key factor in reversing postoperative atelectasis. ${ }^{15}$ The time point of initiation of breathing exercises could be improved if patients were educated and trained before surgery to perform their breathing exercises immediately after surgery, rather than waiting for the first physiotherapy session, which is commonly not provided until the day after surgery. ${ }^{16}$

Preoperative education and breathing exercise training alone is reported to be associated with a $75 \%$ relative risk reduction and absolute risk reduction of $20 \%$ in PPCs, ${ }^{17} 18$ although this effect could be exaggerated by methodological biases of single centre trials, non-masked assessors, and low risk surgical cohorts. Non-reporting of PPC risk factors and non-standardisation of early ambulation and physiotherapy are additional confounders that limit conclusions. Additionally, preoperative education to prevent PPCs has not been tested in the context of recent advances in perioperative management, such as minimally invasive surgery or enhanced recovery after surgery pathways, ${ }^{19}$ or where preoperative education is provided at outpatient clinics many weeks before surgery and by physiotherapists of different experience levels; both confounders of typical current practice at public and private hospitals.

The Lung Infection Prevention Post Surgery Major Abdominal with Pre-Operative Physiotherapy (LIPPSMAck-POP) trial tested the hypothesis that preoperative education and breathing exercise training delivered within six weeks of surgery by physiotherapists reduces the incidence of PPCs after upper abdominal surgery. We tested this pragmatically with physiotherapists of varying levels of experience providing the intervention within existing multidisciplinary preadmission clinics and within the context of modern advances in perioperative management.

\section{Methods}

The trial was a pragmatic, international, multicentre, patient and assessor blinded, parallel group, randomised placebo controlled trial, powered for superiority and conducted at three Australian and New Zealand public hospitals. Site institutional review boards and ethics committees approved the study, and an independent data safety and monitoring board (see appendix) oversaw the trial's safety and ethical conduct. Full details of the trial's rationale, design, protocol, and interventions are published elsewhere. ${ }^{20}$

\section{Participants}

Eligible patients were English speaking adults 18 years or older who were awaiting elective upper abdominal surgery that required general anaesthesia, a minimum overnight hospital stay, and a $5 \mathrm{~cm}$ or longer incision above, or extending above, the umbilicus, and who attended an outpatient preadmission assessment clinic. We excluded patients if they were current hospital inpatients, required organ transplants, required abdominal hernia repairs, were unable to ambulate for more than one minute, and were unable to participate in a single physiotherapy preoperative session within six weeks of surgery. Site investigators screened preadmission clinics daily and invited eligible patients to participate in the trial. Written informed consent was gained before randomisation.

\section{Randomisation}

Preoperative physiotherapists randomly assigned consecutive participants to either intervention (information booklet plus preoperative physiotherapy education and training) or control (information booklet alone) using sequentially numbered sealed opaque envelopes containing allocation cards wrapped in aluminium foil. An independent administrator who took no further part in the trial preprepared these envelopes. Randomisation occurred before the preoperative physiotherapy assessment. Patient details were marked on envelopes to record that randomisation was in order of recruitment. The allocation sequence was determined by a web based computer generated blocked random number table (1:1), which was unavailable to trial staff until completion of the trial.

\section{Masking}

Site investigators and preoperative physiotherapists aware of group allocation had no contact with patients postoperatively. The patients, postoperative physiotherapists, hospital staff, and statisticians were unaware of group assignment. We assessed the success of patient masking in a convenience sample of 29 consecutive participants ${ }^{21}$ (see appendix). Primary and secondary outcome assessors were masked to group allocation and not involved in postoperative clinical management. Data were entered into locked electronic databases. These were unsealed for initial analysis after the final participant had reached the six week follow-up. Databases were resealed until the final 12 month follow-up.

\section{Interventions and procedures}

At participating centres, as per accepted standard care, patients listed for upper abdominal surgery are required to attend a hospital multidisciplinary outpatient clinic for presurgical evaluation within six weeks of surgery. At these clinics patients are seen by a nurse, anaesthetist, doctor, and, if required, a stomal therapist. Consenting eligible patients were entered into the trial and provided with an additional physiotherapy session at these clinics.

The preadmission physiotherapy session for control and intervention participants consisted of a standardised physical and subjective assessment. ${ }^{20}$ The physiotherapist gave participants an information booklet containing written and pictorial information about PPCs and potential prevention with early ambulation and breathing exercises. Within this booklet, breathing exercises were prescribed and consisted of two sets of 10 slow deep breaths followed by three coughs, to be performed hourly and starting 
immediately after surgery. No physiotherapy related information other than that contained within the booklet was provided to control participants. Physicians and nurses at the preadmission clinic provided information to participants at their discretion and this was expected to contain the standard amount of education, information, and preparation from these other professions.

Participants randomised to the intervention group received an additional single 30 minute education and breathing exercise coaching session with the physiotherapist immediately after the standardised physiotherapy assessment and delivery of the booklet. During this session, participants were educated about the possibility of PPCs after surgery and given an individualised risk assessment. ${ }^{7}$ The effect of anaesthesia and abdominal surgery on mucociliary clearance and lung volumes was explained. Consequences of bacterial stagnation in the lungs were highlighted, utilising the booklet's diagram of mucociliary clearance. The participants were informed that although PPCs were preventable with early ambulation it was often not possible to ambulate at the intensity and duration thought to prevent PPCs until the first or second postoperative day. The participants were educated that self directed breathing exercises were vital to protect their lungs during this inactivity phase and to commence them immediately on regaining consciousness and to continue them hourly until fully ambulant. The physiotherapist then trained the intervention participants on how to perform the prescribed breathing exercises, as detailed in the booklet, and they were coached for at least three repetitions. To help patients remember to perform the exercises hourly in the postoperative period, memory cues were provided. Pragmatically, when we were unable to provide interventions face to face, the booklet was mailed to patients and assessment and education were provided by telephone.

Eleven physiotherapists with varying levels of experience provided the preoperative interventions. The physiotherapistsincluded students, new graduates, senior physiotherapists, through to a physiotherapist with 15 years of acute surgical practice and extensive experience in patient education. To ensure consistency in delivery, all physiotherapists viewed an audiovisual recording of the most experienced physiotherapist providing a preoperative intervention and were provided with a semi-scripted guide to the education session.

From the first postoperative day both control and intervention participants received a physiotherapy directed standardised assisted early ambulation programme $^{20}$ (see appendix). Ward physiotherapists assessed the participants daily using standardised criteria $^{22}$ (seeappendix)and discharged the participants from the assisted ambulation service once a threshold score was met. At the first ambulation session, ward physiotherapists provided participants with a walking aid if needed, an abdominal support pillow for use during coughing, and a brief reminder to perform the breathing exercises as described within the information booklet provided preoperatively. Other than the daily assisted ambulation programme and the brief breathing exercise reminder on the first postoperative day, no additional respiratory physiotherapy was provided to either control or intervention participants. Site investigators monitored and reported divergence from this protocol. If nursing staff provided respiratory devices (eg, incentive spirometry or positive expiratory pressure devices), site investigators removed these and recorded the incidence (see appendix). If a participant was diagnosed as having the primary PPC endpoint, a site investigator informed the ward physiotherapist, and respiratory physiotherapy was subsequently delivered at the attending physiotherapist's discretion. No attempt was made to standardise the way medical or nursing staff encouraged participants to perform breathing exercises as this was considered unfeasible and not reflective of pragmatic ward practice. All other aspects of perioperative patient care, including the type of anaesthesia, postoperative analgesia, surgical techniques, and postoperative clinical care were provided at the discretion of the anaesthesia and surgical teams and according to routine clinical practice at each centre.

\section{Outcome measures}

The primary outcome was incidence of a PPC within 14 postoperative days, or hospital discharge, whichever came sooner. Assessors masked to group allocation assessed participants prospectively and daily until the seventh postoperative day. From the seventh postoperative day additional assessments were performed only as clinically suspected until day 14 when signs or symptoms of respiratory system deterioration were reported in the medical record. Participants were screened using a standardised validated diagnostic tool ${ }^{7-10} 1820$ consisting of eight symptomatic and diagnostic criteria (see box 1). A PPC was diagnosed when four or more of these eight criteria were present at any time from midnight to midnight each postoperative day.

Secondary outcomes included pneumonia, ${ }^{23}$ defined as the presence of new chest infiltrates on radiography with at least two of the following criteria: temperature $>38^{\circ} \mathrm{C}$, dyspnoea, cough and purulent sputum, altered respiratory auscultation, and leukocytosis $>14000 / \mathrm{mL}$ or leucopenia $<3000 / \mathrm{mL}$ within the first 14 hospital days, length of hospital stay (acute and subacute inclusive), readiness for hospital discharge ${ }^{24}$ within the first 21 hospital days, number of days in an intensive care or high dependency unit, all cause unplanned admissions to an intensive care or high dependency unit, and hospital costs. Additional secondary outcomes measured at six weeks were self reported health related quality of life and physical function using the SF-36 version $2^{25}$ and specific activity questionnaire, ${ }^{26}$ hospital readmissions, and self reported complications that required medical review (respiratory, thromboembolic event, cardiac, gastrointestinal, wound infection, fatigue, or 
Box 1: Postoperative pulmonary complication diagnostic tool: Melbourne group score

Diagnosis confirmed when four or more criteria are present in a postoperative day:

- New abnormal breath sounds on auscultation different from in the preoperative assessment

- Production of yellow or green sputum different from in the preoperative assessment

- Pulse oximetry oxygen saturation $\left(\mathrm{SpO}_{2}\right)<90 \%$ on room air on more than one consecutive postoperative day

- Maximum oral temperature $38^{\circ} \mathrm{C}$ on more than one consecutive postoperative day

- Chest radiography report of collapse or consolidation

- An unexplained white cell count greater than $11 \times 10^{9} / \mathrm{L}$

- Presence of infection on sputum culture report

- Physician's diagnosis of pneumonia, lower or upper respiratory tract infection, an undefined chest infection, or prescription of an antibiotic for a respiratory infection

weakness). Following newly published meta-analysis data showing a strong association between mortality and PPCs, ${ }^{4}$ we added a further secondary outcome of 12 month all cause mortality one year into the trial. Assessors masked to group assignment retrieved these data for all participants from government databases.

To assess standardisation of postoperative ambulation we measured hours from surgery until participants were ambulant with a physiotherapist for longer than one minute, days until ambulant for longer than 10 minutes, and days until discharged from assisted ambulation.

\section{Statistical analysis}

Sample size

The study was powered based on two rationales: absolute risk reduction in PPCs of $20 \%$ as reported by previous trials of preoperative education, ${ }^{17} 18$ and a PPC rate of $38 \%$ (95\% confidence interval $26 \%$ to $52 \%$ ) at the primary participating institution identified by retrospective audit of consecutive patients requiring upper abdominal surgery ( $\mathrm{n}=50$, unpublished data, 2008). For the purposes of this trial, conservative goals (minimum 10\% absolute risk reduction from a $20 \%$ baseline PPC risk) were set considering time passed since previous audits and trials, known improvements in perioperative care during this time, and methodological limitations of previous research. A priori we estimated a sample of 398 patients would have $80 \%$ power to detect a significant difference between groups ( $\mathrm{P}=0.05$, two sided) with an $11 \%$ inflation to account for drop-outs, non-compliance, and uncertainty of baseline risk, providing a final sample size of 441 .

\section{Baseline comparability and adjustment factors}

Results were adjusted using backwards stepwise regression for specific baseline covariates considered a priori $^{20}$ to affect primary outcome. These prespecified covariates were respiratory comorbidity, smoking history, physical activity, age, obesity, duration of operation, surgical category, incision type, admission to intensive care, intraoperative ventilation, fluid delivery, blood transfusions, postoperative analgesia mode, and prophylactic antibiotics.

\section{Analysis of primary and secondary outcomes}

To estimate primary outcome efficacy and binomial secondary outcomes we used multivariate robust random effects Poisson generalised linear regression. We compared the time effect of day the PPC was diagnosed (to day 14) and mortality (to 12 months) between groups using Cox proportional hazards regression with or without adjustment for covariates and graphically illustrated using Kaplan-Meier methods. Analysis of hospital length of stay and readiness for hospital discharge (to 21 days) was prespecified ${ }^{20}$ using mixed effects ordered logistic regression. However, as these time points are truncated in patients who died, we also performed a sensitivity analysis using Cox proportional hazards regression with or without adjustment for covariates, where deaths were treated as censored times without failure.

For all outcomes we estimated differences in effect size between groups on an intention-to-treat basis. We recruited patients with an anticipated surgical procedure complying with the trial protocol. At times this planned procedure was changed intraoperatively to lower abdominal or laparoscopic surgery. We also performed a prespecified per protocol analysis excluding participants operated on through an incision wholly below the umbilicus or by laparoscope alone. ${ }^{20}$ These participants were not provided with assisted ambulation physiotherapy as this was not standard care at participating sites for this patient cohort. In these participants we therefore did not assess days to discharge from assisted ambulation. We excluded from all analyses those participants who failed to progress to surgery or withdrew their consent.

\section{Exploratory analyses}

We performed exploratory post hoc sensitivity adjusted analyses of the per protocol population to determine the effect of specific covariates (experience grade of treating physiotherapist-experience less than five years versus experience more than five years; surgical group-upper gastrointestinal/ hepatobiliary, colorectal, renal/urology, preoperative respiratory complication risk score, ${ }^{7}$ age, and sex) across all primary and major secondary outcomes. These covariates were selected to assist in hypothesis generation according to known factors influencing the incidence of PPCs and the successful provision of an education based intervention.

The statistical analysis plan was prespecified ${ }^{20}$ and we used STATA (version 14.1) for all analyses.

\section{Patient involvement}

Qualitative studies report that patients rate preoperative counselling and the avoidance of infection as the two most important strategies for improving recovery after upper abdominal surgery, ${ }^{27}$ preferring personalised delivery of detailed information. ${ }^{28}$ This meets patients' need for control over their disease and surgery. ${ }^{29}$ However, health professionals tend to underestimate these factors. ${ }^{30}$ Before designing the current study, the corresponding author invited patients who had 


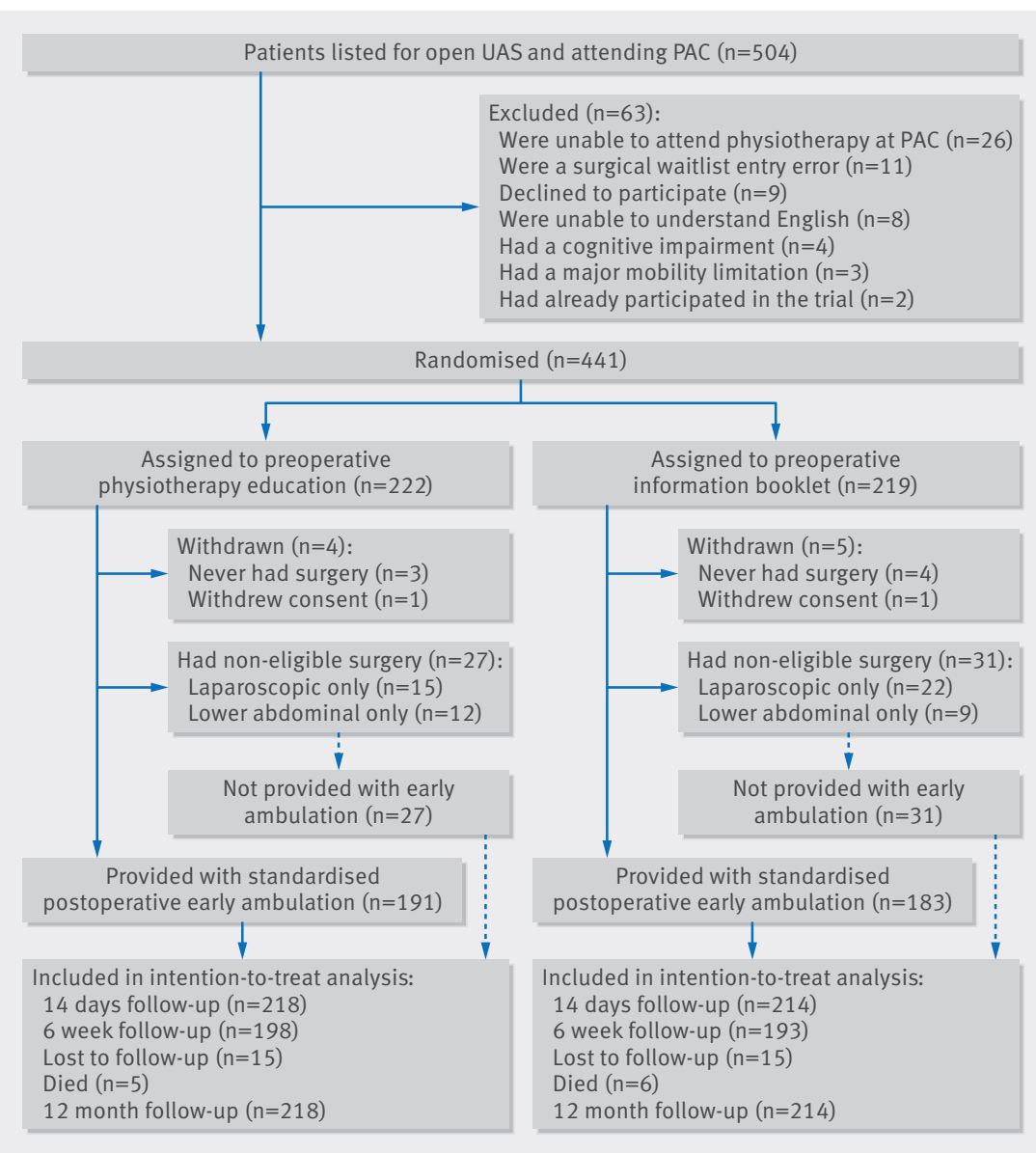

Fig 1 | Flow of patients through trial. UAS=upper abdominal surgery. $\mathrm{PAC}=$ preadmission clinic patients were withdrawn from the trial, leaving 432 (98\%) included for primary analysis (fig 1). Tables 1 and 2 list the baseline and clinical characteristics of the participants.

\section{Primary outcome}

Overall, 85 of the 432 participants (20\%) were diagnosed as having a PPC. Intention-to-treat unadjusted results showed statistically significantly fewer PPCs in the physiotherapy group (27/218, $12 \%$ ) compared with control group (58/214, 27\%); (absolute risk reduction 15\%, 95\% confidence interval $7 \%$ to $22 \%, \mathrm{P}<0.001$; table 3$)$. The incidence of PPCs remained halved (hazard ratio $0.48,95 \%$ confidence interval 0.30 to $0.75, \mathrm{P}=0.001$ ) in the intervention group when adjustments were made for baseline imbalances in three of the prespecified covariatesage, respiratory comorbidity, surgical procedure (table 3, fig 2), with a number needed to treat of 7 (95\% confidence interval 5 to 14 ).

\section{Secondary outcomes}

The incidence of hospital acquired pneumonia was halved in the physiotherapy group in the adjusted analyses (table 3), with a number needed to treat of 9 (95\% confidence interval 6 to 21). No differences were detected in the other secondary measures of hospital length of stay, readiness for hospital discharge, unplanned readmissions or length of stay in intensive care, hospital readmissions at six weeks, and all ambulation attainment measures (table 3 and appendix). No adverse events were attributable to the preoperative physiotherapy education sessions or to the assisted ambulation protocol. Detailed modelling of specific costs and health economics supporting this clinical efficacy report will be published later.

Five participants (1\%) died during the primary hospital stay. Four participants (two each in both groups) acquired a PPC in the first three postoperative days, progressing to respiratory sepsis, multi-organ failure, and then death. The fifth death occurred in a participant who developed a PPC on the 11th postoperative day and later died of a thromboembolic event. A PPC within the first 14 postoperative days was associated with increased mortality at all time points after surgery (unadjusted 12 month mortality: 24\% (20/85) in participants with PPCs $v 6 \%$ (20/347) without PPCs; $\mathrm{P}<0.001$; adjusted data figure $1 \mathrm{~S}$ : appendix). No difference in all cause mortality between groups was seen at six weeks and 12 months, although a sustained separation between groups favouring the intervention group starting at four months was evident (adjusted hazard ratio 0.78, 95\% confidence interval 0.41 to $1.48, \mathrm{P}=0.45$; fig $3 \mathrm{a}$ ).

\section{Sensitivity analyses}

Planned per protocol sensitivity analysis removing participants who had lower abdominal and laparoscopic surgery found strengthening of effect in the primary and most secondary outcomes in favour of physiotherapy (see appendix). After surgery, 15 (3\%) 


\begin{tabular}{|c|c|c|}
\hline Characteristics & Preoperative physiotherapy $(n=218)$ & Information booklet $(n=214)$ \\
\hline Median (interquartile range) age (years) & $63.4(51.5-71.9)$ & $67.5(56.3-75.3)$ \\
\hline Men & $132(61)$ & $134(61)$ \\
\hline Mean (SD) body mass index & $28.5(5.9)$ & $28.3(6.2)$ \\
\hline Body mass index $>35$ & $25(12)$ & $30(14)$ \\
\hline \multicolumn{3}{|l|}{ ASA physical health status: } \\
\hline $1-2$ & $150(69)$ & $124(58)$ \\
\hline $3-4$ & $67(31)$ & $90(42)$ \\
\hline \multicolumn{3}{|l|}{ Comorbidities: } \\
\hline Respiratory disease & $42(19)$ & $55(26)$ \\
\hline Diabetes mellitus & $33(15)$ & $41(19)$ \\
\hline Cancer & $148(68)$ & $148(69)$ \\
\hline Cardiac disease & $26(12)$ & $34(16)$ \\
\hline Median (interquartile range) functional comorbidity index & $2(1-5)$ & $2(1-4)$ \\
\hline \multicolumn{3}{|l|}{ Preoperative respiratory status } \\
\hline \multicolumn{3}{|l|}{ Chronic daily sputum typology: } \\
\hline Mucoid & $44(20)$ & $46(22)$ \\
\hline Mucopurulent or purulent & $28(13)$ & $20(9)$ \\
\hline Recent chest infection* & $12(6)$ & $5(2)$ \\
\hline \multicolumn{3}{|l|}{ Smoking status: } \\
\hline Never smoked & $76(35)$ & $71(33)$ \\
\hline Former smokert & $93(43)$ & $86(40)$ \\
\hline Current smoker & $49(21)$ & $57(27)$ \\
\hline Mean (SD) average pack years & $18.1(23.7)$ & $20.6(24.7)$ \\
\hline \multicolumn{3}{|l|}{ Preoperative strength and activity levels } \\
\hline Mean (SD) handgrip strength $(\mathrm{kg})$ & $35.1(11.4)$ & $33.8(10.9)$ \\
\hline Mean (SD) estimated $\mathrm{VO}_{2} \max \S(\mathrm{mL} / \mathrm{kg} / \mathrm{min})$ & $16.1(6.9)$ & $15.3(7.0)$ \\
\hline Mean (SD) self reported maximum METS§ & $6.5(2.0)$ & $6.3(2.1)$ \\
\hline \multicolumn{3}{|l|}{ Provision of interventions } \\
\hline \multicolumn{3}{|l|}{ Physiotherapist experience grade: } \\
\hline Student & $31(14)$ & $30(14)$ \\
\hline Recent graduate & $47(22)$ & $54(25)$ \\
\hline Senior & $16(7)$ & $11(5)$ \\
\hline Specialist respiratory & $124(57)$ & $119(56)$ \\
\hline Provided by telephone & $18(8)$ & $24(11)$ \\
\hline Median (interquartile range) days from preoperative physiotherapy to surgery & $8(3-16)$ & $9(4-20)$ \\
\hline \multicolumn{3}{|l|}{ Surgical category and procedure } \\
\hline Colorectal: & $108(50)$ & $101(47)$ \\
\hline Hemicolectomy & $33(15)$ & $37(17)$ \\
\hline Anterior and anteroposterior resection & $33(15)$ & $36(17)$ \\
\hline Hartmann's (including reversals) & $13(6)$ & $9(4)$ \\
\hline Other bowel resections & $29(13)$ & $19(9)$ \\
\hline Hepatobiliary/upper gastrointestinal: & $49(22)$ & $59(28)$ \\
\hline Oesophagectomy/gastrectomy & $12(6)$ & $21(10)$ \\
\hline Liver surgery & $17(8)$ & $14(7)$ \\
\hline Whipples/pancreadectomy & $13(6)$ & $12(6)$ \\
\hline Other & $7(3)$ & $12(6)$ \\
\hline Renal/urology/other: & $61(28)$ & $54(24)$ \\
\hline Nephrectomy & $35(16)$ & $31(14)$ \\
\hline Cystoprostatectomy/cystectomy & $9(4)$ & $7(3)$ \\
\hline Adrenalectomy/pyeloplasty & $5(2)$ & $9(4)$ \\
\hline Other & $12(6)$ & $7(3)$ \\
\hline \multicolumn{3}{|l|}{ Incision type: } \\
\hline Midline laparotomy & $109(50)$ & $103(48)$ \\
\hline Bilateral or unilateral subcostal & $40(18)$ & $38(18)$ \\
\hline Transverse abdominal & $33(15)$ & $34(16)$ \\
\hline Abdominal+thoracotomy & $6(3)$ & $7(3)$ \\
\hline Other upper abdominal incision & $3(1)$ & $1(0)$ \\
\hline Laparoscopic or lower abdominal & $27(12)$ & $31(14)$ \\
\hline \multicolumn{3}{|l|}{ Length of procedure (mins): } \\
\hline$<120$ & $21(10)$ & $15(7)$ \\
\hline $120-179$ & $40(18)$ & $40(19)$ \\
\hline $180-239$ & $62(28)$ & $64(30)$ \\
\hline $240-299$ & $37(17)$ & $36(17)$ \\
\hline
\end{tabular}


Table 1 | (Continued)

Characteristics

Preoperative physiotherapy $(n=218)$

Information booklet $(\mathrm{n}=214)$

$\geq 300$

$58(27)$

$59(28)$

Intraoperative management

Mechanical ventilation:

\begin{tabular}{|c|c|c|}
\hline Mean (SD) F,02 & $0.54(0.14)$ & $0.56(0.13$ \\
\hline Median (interquartile range) PEEP $\left(\mathrm{cm} \mathrm{H}_{2} \mathrm{O}\right)$ & $5(5-6)$ & $5(4-6)$ \\
\hline Mean (SD) tidal volume (mL/kg) & $6.2(1.3)$ & $6.2(1.3)$ \\
\hline Mean (SD) intraoperative fluid delivery (mL/kg/hr) & $9.1(4.5)$ & $9.0(4.6)$ \\
\hline \multicolumn{3}{|l|}{ Type of intraoperative fluid: } \\
\hline Crystalloid & $213(98)$ & $202(94)$ \\
\hline Colloid & $5(2)$ & $1(0)$ \\
\hline \multicolumn{3}{|l|}{ No of transfusion units: } \\
\hline $1-2$ & $7(3)$ & $6(3)$ \\
\hline$>2$ & $2(1)$ & $4(2)$ \\
\hline Intraoperative epidural & $47(22)$ & $44(21)$ \\
\hline \multicolumn{3}{|l|}{ Postoperative management } \\
\hline \multicolumn{3}{|l|}{ Immediate postoperative location: } \\
\hline Surgical ward & $124(57)$ & $115(54)$ \\
\hline ICU & $94(43)$ & $99(46)$ \\
\hline ICU with mechanical ventilation & $21(10)$ & $23(11)$ \\
\hline Mean (SD) fluid delivery postoperative day $1(\mathrm{~mL} / \mathrm{kg} / \mathrm{min})$ & $1.4(0.8)$ & $1.5(1.1)$ \\
\hline \multicolumn{3}{|c|}{ Antibiotic delivery before PPC, discharge from hospital, or day 14 , whichever came first: } \\
\hline Prophylactic during surgery & $212(97)$ & $209(98)$ \\
\hline All types of $\beta$ lactamase inhibitor & $191(88)$ & $183(86)$ \\
\hline Penicillins and 1st-2nd generation cephalosporins & $186(85)$ & $180(84)$ \\
\hline 3rd generation cephalosporins/macrolides & $16(7)$ & $20(9)$ \\
\hline Other & $33(15)$ & $35(16)$ \\
\hline \multicolumn{3}{|l|}{ Analgesia management: } \\
\hline Oral & $210(96)$ & 211 (99) \\
\hline Patient controlled intravenous & $159(73)$ & $178(83)$ \\
\hline Patient controlled epidural & $22(10)$ & $20(9)$ \\
\hline Continuous epidural & $45(21)$ & $38(18)$ \\
\hline Continuous infusion pump & $26(12)$ & $26(12)$ \\
\hline
\end{tabular}

ASA=American Society Anaesthesiologists score where 1 is a normal healthy patient, 2 is a patient with mild systemic disease, 3 is a patient with severe systemic disease, 4 is a patient with severe systemic disease that is a constant threat to life, and 5 is a moribund patient who is not expected to survive; VO2=rate of oxygen consumption; METS=metabolic equivalents; FiO2=Fraction of inspired oxygen; PEEP=positive end expiratory pressure; ICU=intensive care unit; PPC=postoperative pulmonary complications.

*Reported cough with new yellow/green sputum and symptoms of malaise, fever, or dyspnoea within two weeks of preoperative assessment.

tCeased smoking more than eight weeks before preoperative assessment.

¥Smoked tobacco regularly within eight weeks of assessment.

§Derived from specific activity questionnaire.

Table 2 | Postoperative clinical events and complications between groups

No (\%)

Clinical events or complications

Preoperative physiotherapy $(n=218)$

Information booklet $(\mathrm{n}=\mathbf{2 1 4})$

Blood volume complications:

\begin{tabular}{|c|c|c|}
\hline Hypovolemia & $26(12)$ & $26(12)$ \\
\hline Fluid overload & $7(3)$ & $4(2)$ \\
\hline Surgical lacerations & $22(10)$ & $12(6)$ \\
\hline Wound dehiscence & $5(2)$ & $8(4)$ \\
\hline Anastomosis leaks & $3(1)$ & $2(1)$ \\
\hline \multicolumn{3}{|l|}{ fection type: } \\
\hline Wound & $22(10)$ & $25(10)$ \\
\hline Sepsis & $8(4)$ & $14(7)$ \\
\hline \multicolumn{3}{|l|}{ ther events: } \\
\hline Delirium & $17(8)$ & $22(10)$ \\
\hline Re-intubation & $8(4)$ & $11(5)$ \\
\hline Cardiac event & $11(5)$ & $7(3)$ \\
\hline Fall & $0(0)$ & $2(1)$ \\
\hline Acute respiratory failure & $5(2)$ & $12(6)$ \\
\hline
\end{tabular}




\begin{tabular}{|c|c|c|c|c|c|c|}
\hline \multirow[b]{2}{*}{ Intention to treat } & \multirow{2}{*}{$\begin{array}{l}\text { Preoperative } \\
\text { physiotherapy } \\
(n=218)\end{array}$} & \multirow[b]{2}{*}{$\begin{array}{l}\text { Information } \\
\text { booklet } \\
(n=214)\end{array}$} & \multicolumn{2}{|l|}{ Adjusted analysis } & \multicolumn{2}{|l|}{ Unadjusted analysis } \\
\hline & & & $\begin{array}{l}H R, O R, I R R \\
(95 \% \mathrm{Cl})\end{array}$ & $P$ value & $\begin{array}{l}H R, \text { OR, IRR } \\
(95 \% \mathrm{Cl})\end{array}$ & $P$ value \\
\hline \multicolumn{7}{|l|}{ Primary outcome } \\
\hline PPC & $27(12)$ & $58(27)$ & $0.48(0.30$ to 0.75$)$ & 0.001 & $0.43(0.27$ to 0.67$)$ & $<0.001$ \\
\hline \multicolumn{7}{|l|}{ Secondary outcomes } \\
\hline Pneumonia & $18(8)$ & $42(20)$ & $0.45(0.26$ to 0.78$)$ & 0.005 & $0.40(0.23$ to 0.69$)$ & $<0.001$ \\
\hline \multicolumn{7}{|l|}{ Hospital utilisation: } \\
\hline Median (interquartile range) length of hospital stay (days) ${ }^{\star}$ & $8(6-11)$ & $9(7-13)$ & 0.85 (0.61 to 1.19$)$ & 0.35 & 0.77 (0.56 to 1.07$)$ & 0.13 \\
\hline Sensitivity analysis $\dagger$ & & & $1.12(0.94$ to 1.34$)$ & 0.22 & $1.19(0.99$ to 1.43$)$ & 0.06 \\
\hline \multicolumn{7}{|l|}{ Ready for hospital discharge (days): } \\
\hline Median (interquartile range) ${ }^{\star}$ & $6(5-10)$ & $7(5-11)$ & 0.85 (0.61 to 1.18$)$ & 0.33 & 0.77 (0.56 to 1.08$)$ & 0.13 \\
\hline Sensitivity analysis ${ }^{\prime}$ & & & $1.07(0.90$ to 1.28$)$ & 0.45 & 1.15 (0.96 to 1.38$)$ & 0.14 \\
\hline Median (interquartile range) ICU length of stay (days) & $1.3(2.9)$ & $1.5(2.7)$ & 0.97 (0.67 to 1.42$)$ & 0.89 & $0.83(0.57$ to 1.18$)$ & 0.29 \\
\hline Unplanned ICU readmissions & $15(7)$ & $19(9)$ & $0.93(0.47$ to 1.85$)$ & 0.84 & 0.78 (0.39 to 1.53$)$ & 0.46 \\
\hline Hospital readmission at six weeks & 36/197 (18) & 33/199 (17) & $1.14(0.71$ to 1.84$)$ & 0.59 & $1.10(0.69$ to 1.77$)$ & 0.69 \\
\hline \multicolumn{7}{|l|}{ Mobilitył: } \\
\hline $\begin{array}{l}\text { Median (interquartile range) time from operation to ambulation } \\
>1 \text { min (hours) }\end{array}$ & $23(20-44)$ & $22(20-39)$ & $1.03(0.82$ to 1.30$)$ & 0.81 & $1.08(0.86$ to 1.37$)$ & 0.50 \\
\hline $\begin{array}{l}\text { Median (interquartile range) postoperative day achieved >10 } \\
\text { mins of ambulation (days) }\end{array}$ & $3(1-5)$ & $3(1-5)$ & 0.99 (0.83 to 1.17) & 0.90 & $1.05(0.88$ to 1.25$)$ & 0.58 \\
\hline $\begin{array}{l}\text { Median (interquartile range) postoperative day discharged from } \\
\text { assisted ambulation (days) }\end{array}$ & $3(2-5)$ & $4(2-5)$ & 1.05 (0.86 to 1.28$)$ & 0.60 & $1.14(0.94$ to 1.39$)$ & 0.19 \\
\hline \multicolumn{7}{|l|}{ Patient reported complications at 6 weeks: } \\
\hline Any complications & 74/192(39) & $79 / 197(40)$ & $0.90(0.65$ to 1.24$)$ & 0.50 & 0.89 (0.65 to 1.22$)$ & 0.65 \\
\hline Wound infection & 36/192 (19) & 40/197(20) & 0.88 (0.56 to 1.39$)$ & 0.50 & $0.92(0.59$ to 1.45$)$ & 0.73 \\
\hline Fatigue & 29/192 (14) & 33/197 (14) & 1.00 (0.61 to 1.66$)$ & 0.99 & $0.90(0.55$ to 1.48$)$ & 0.65 \\
\hline Nausea/vomiting/gastrointestinal & $27 / 192(14)$ & 29/197 (14) & $0.98(0.58$ to 1.67$)$ & 0.94 & $0.96(0.57$ to 1.61$)$ & 0.86 \\
\hline Respiratory & $8 / 192(4)$ & $21 / 197(9)$ & 0.45 (0.20 to 1.03$)$ & 0.059 & 0.39 (0.17 to 0.88$)$ & 0.024 \\
\hline Cardiac & 10/192(5) & $3 / 197(2)$ & $4.06(1.09$ to 15.1$)$ & 0.036 & $3.42(0.94$ to 12.4$)$ & 0.062 \\
\hline Venothromboembolic events & $2 / 192(1)$ & 6/197 (3) & $0.37(0.07$ to 1.85$)$ & 0.23 & $0.34(0.07$ to 1.69$)$ & 0.19 \\
\hline \multicolumn{7}{|l|}{ Mean (SD) mortality: } \\
\hline In hospital & $3(1.4)$ & $3(1.4)$ & 1.72 (0.42 to 7.01$)$ & 0.45 & $1.25(0.26$ to 5.96$)$ & 0.78 \\
\hline At 6 weeks & $4(1.8)$ & $3(1.4)$ & $1.47(0.32$ to 6.72$)$ & 0.62 & $1.31(0.29$ to 5.83$)$ & 0.72 \\
\hline At 12 months & $16(7.3)$ & $23(11)$ & 0.78 (0.41 to 1.48$)$ & 0.45 & $0.67(0.35$ to 1.27$)$ & 0.22 \\
\hline
\end{tabular}

$\mathrm{PPC}=$ postoperative pulmonary complication; $\mathrm{ICU}=$ intensive care unit: $\mathrm{HR}=$ hazard ratio; $\mathrm{OR}=$ odds ratio; $\mathrm{IRR}=$ incidence rate ratio.

Analyses are adjusted for baseline age, previous respiratory disease, and hepatobiliary/upper gastrointestinal surgery. Point estimates are HRs for all outcomes except for ORs for prespecified analysis of hospital length of stay and readiness to discharge, unplanned admission to an ICU, length of stay on an ICU, and hospital readmissions at six weeks, and IRRs for patient reported complications.

*Prespecified analysis involved a rank ordered comparison of length of stay (days), using mixed effects ordered logistic regression. OR $<1.00$ indicates an earlier discharge from hospital.

TTime-to-event analysis with median (interquartile range) number of days reported and estimation of HR using Cox proportion hazards regression. HR $>1.00$ indicates an increased likelihood of earlier discharge from hospital.

‡No mobility measures are available for patients who did not have upper abdominal surgery.

breaches to the postoperative protocol occurred (see appendix). Removal of these patients from analysis did not affect the reduction in PPCs (hazard ratio 0.48, 95\% confidence interval 0.3 to 0.7 ). To explore variations of effect and to validate the main results, we performed further exploratory post hoc adjusted analyses of subgroup effects (experience level of preoperative physiotherapist, site, and participant age, sex, surgical category, and predicted PPC risk score) in PPCs, hospital stay, and 12 month mortality. There was a gradient in PPC reduction according to surgical category, with the greatest response to preoperative physiotherapy in colorectal surgery, then upper gastrointestinal surgery, with the least difference between groups for urology (fig 4). A similar pattern according to type of surgery was seen with length of stay and mortality (fig 5 and fig 6). PPC reduction attributable to the preoperative intervention was greatest in participants educated by an experienced physiotherapist, men, and those younger than 65 years (fig 4). In particular, education

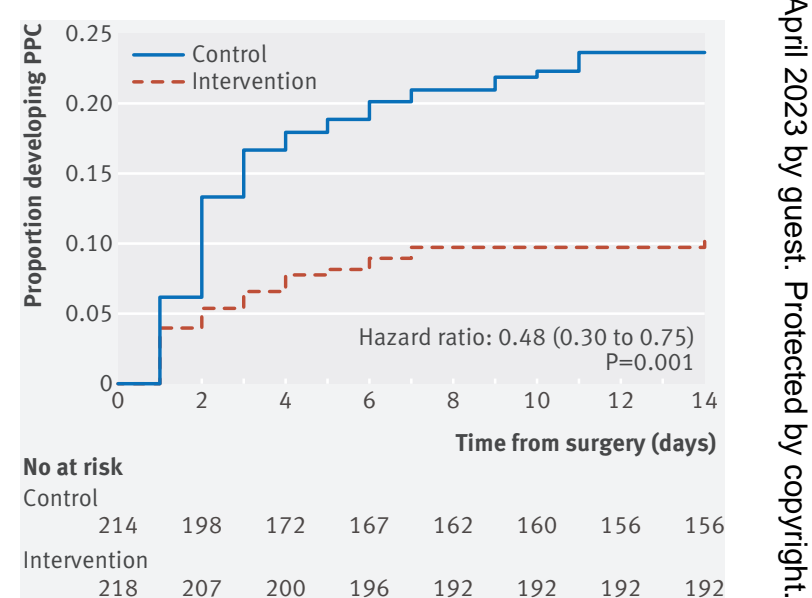

Fig 2 | Time to diagnosis of a postoperative pulmonary complication after surgery. Data are on an intention-totreat basis and adjusted for age, previous respiratory disease, and surgical category. PPC=postoperative pulmonary complication 

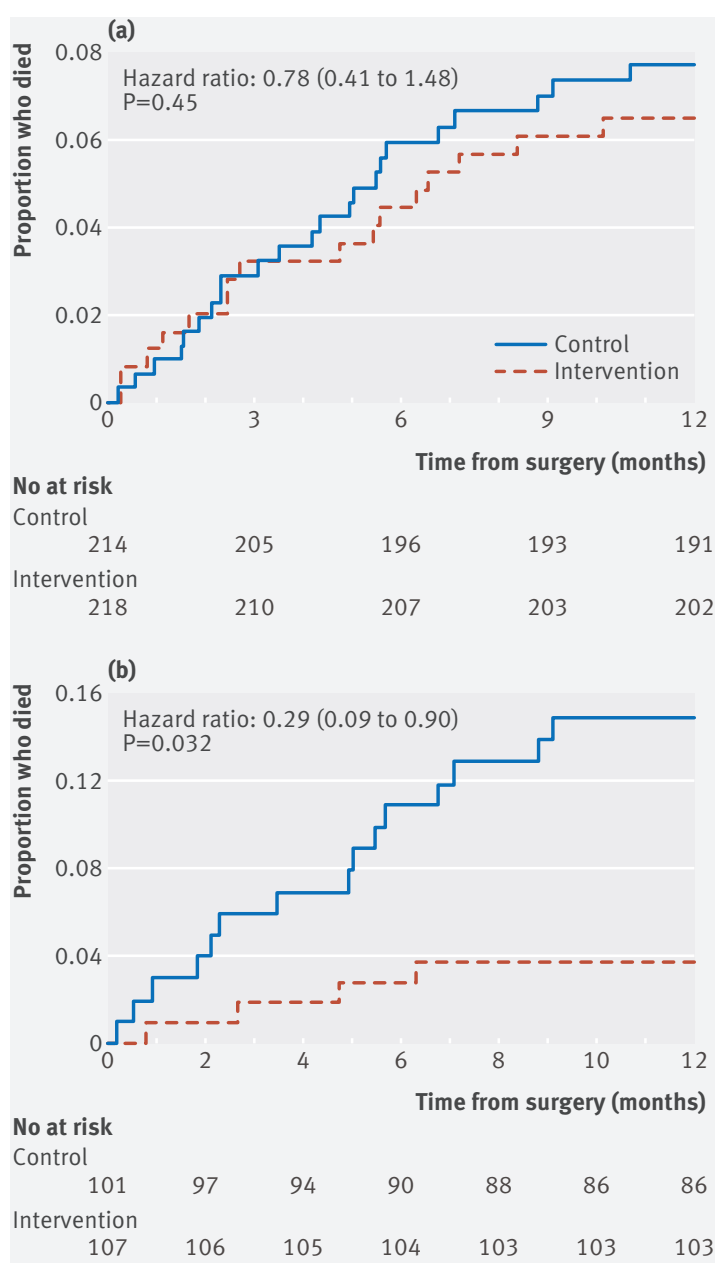

Fig 3 | (a) 12 month mortality between groups; (b) 12 month mortality between groups in subgroup treated by experienced physiotherapists. Data are per protocol and adjusted for age, previous respiratory disease, and surgical category

provided by experienced physiotherapists was associated with shorter length of stay (fig 5) and lower all cause 12 month mortality (adjusted hazard ratio $0.29,95 \%$ confidence interval 0.09 to $0.90, \mathrm{P}=0.032$; fig 3b).

At the New Zealand site, the reduction in PPCs was less than at Australian sites. Exploratory between site covariate analysis found that the New Zealand site provided fewer interventions with experienced physiotherapists $(0 \% v 68 \%, \mathrm{P}<0.001)$, less intraoperative fluid (mean $5.1 \vee 9.8 \mathrm{~mL} / \mathrm{kg} / \mathrm{hr}$, $\mathrm{P}<0.001)$, more epidurals (50\% v12\%, $\mathrm{P}<0.001)$, and later commencement of postoperative ambulation (52 hours $v 28$ hours, $\mathrm{P}<0.001)$. There were no statistically significant differences between sites in the proportion of participants who had colorectal surgery, were male, or were younger than 65 years.

\section{Discussion}

In this multicentre trial conducted in two countries we found that a single 30 minute face-to-face preoperative physiotherapy education and training session provided within six weeks of surgery halved the incidence of postoperative pulmonary complications (PPCs), including hospital acquired pneumonia, after major upper abdominal surgery compared with information provided by a booklet alone. This association was stronger in patients having colorectal surgery, those younger than 65 years, men, or where an experienced physiotherapist provided the education.

\section{Hypothesis of effect}

Atelectasis is inevitable in the early postoperative period because of the pathophysiological effects of anaesthesia, ${ }^{31}$ mechanical ventilation, ${ }^{32}$ and changes in thoracoabdominal pressure. ${ }^{13}$ Postoperative breathing exercises performed by patients might reverse this atelectasis, although breathing exercises coached by physiotherapists postoperatively appear less effective in reducing $\mathrm{PPCs}^{14}$ compared with preoperative interventions. ${ }^{17} 18$ 33-35 One explanation for the effectiveness of preoperative physiotherapy to reduce PPCs is that the preparation, motivation, and training of patients before surgery brings the timing of breathing exercise initiation forward to immediately after regaining consciousness after surgery. Commonly in a postoperative only physiotherapy service, coaching begins on the first or second postoperative day ${ }^{15}$; which may be too late, as most PPCs have already occurred by this time. ${ }^{48}$ Timing of initiation could be critical. Breathing exercises during the first 24 hours after surgery could prevent mild atelectasis extending to severe atelectasis, at which point breathing exercises are less effective in re-expanding non-compliant collapsed lung tissue. ${ }^{15}$ Earlier initiation may also increase the total dose of breathing exercises. Pain, nausea, analgesia, anxiety, and persisting sedation can also compromise a patient's ability to comprehend instructions when first contact with physiotherapy is only in the postoperative phase. In this trial, a sample of intervention patients reported that preoperative physiotherapy education was memorable and engaging. ${ }^{21}$ These patients reported that preoperative physiotherapy empowered them to treat themselves and placed high value on its role in improving their postoperative recovery. ${ }^{21}$

\section{Comparison with other studies}

Our PPC reduction of an adjusted 52\% relative risk reduction is less than that reported in methodologically weaker trials with limitations on generalisability. ${ }^{17} 18$ A Pakistani trial ${ }^{18}$ of 224 patients who were young (mean age 37), having minor surgeries, and of a reasonably healthy premorbid status, reported that preoperative education by medical registrars resulted in earlier postoperative mobilisation and a 76\% relative reduction in PPCs. Similarly, a single centre Swedish trial of 368 patients ${ }^{17}$ reported a $78 \%$ PPC risk reduction after open abdominal surgery where participants were met by experienced physiotherapists the day before surgery, taught postoperative breathing exercises, and educated about early ambulation. Despite the large effect sizes, the generalisability 


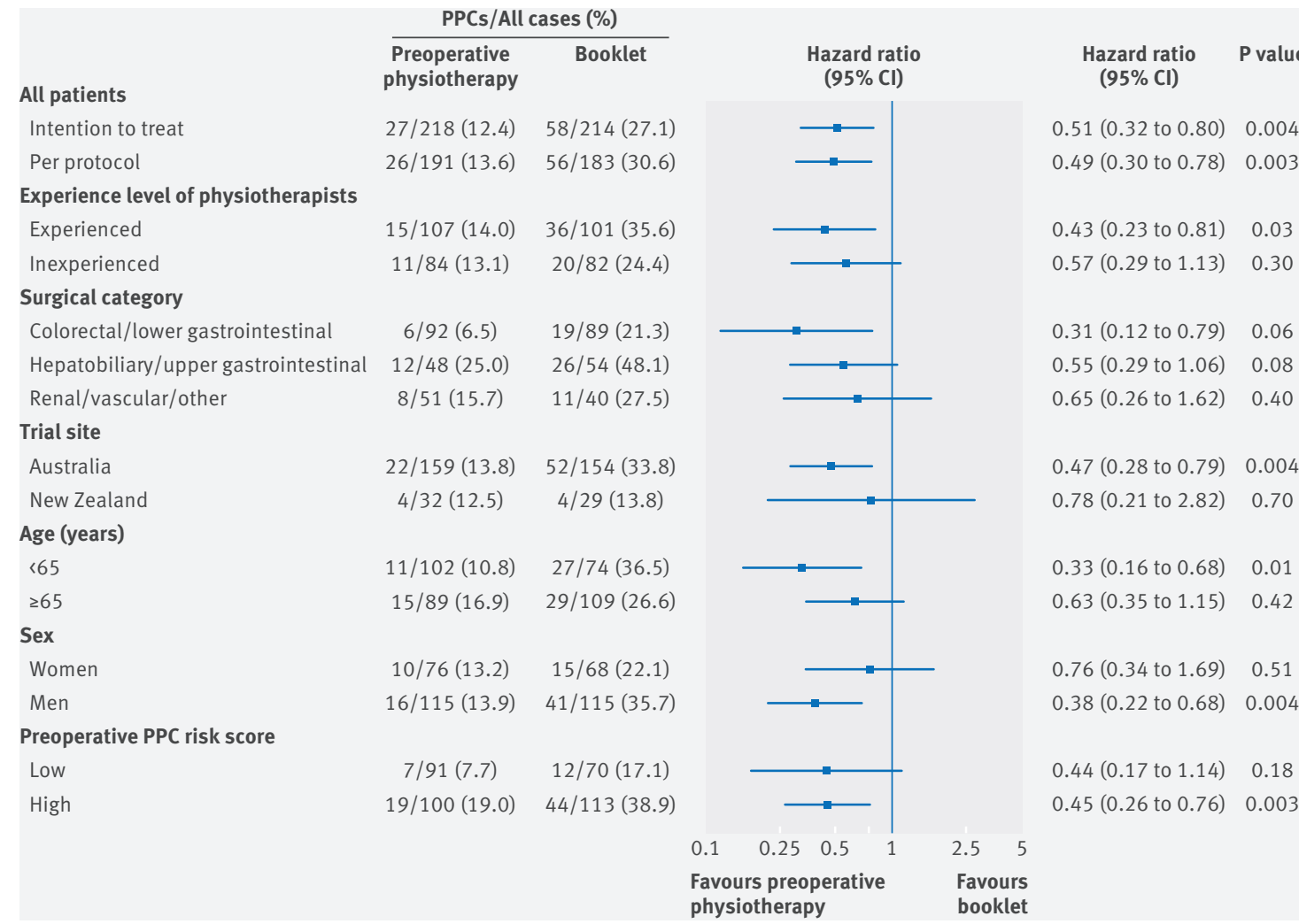

Fig 4 | Sensitivity analysis of subgroup effects on incidence of postoperative pulmonary complications (PPCs). Data are adjusted for age, respiratory comorbidity, and upper gastrointestinal surgery

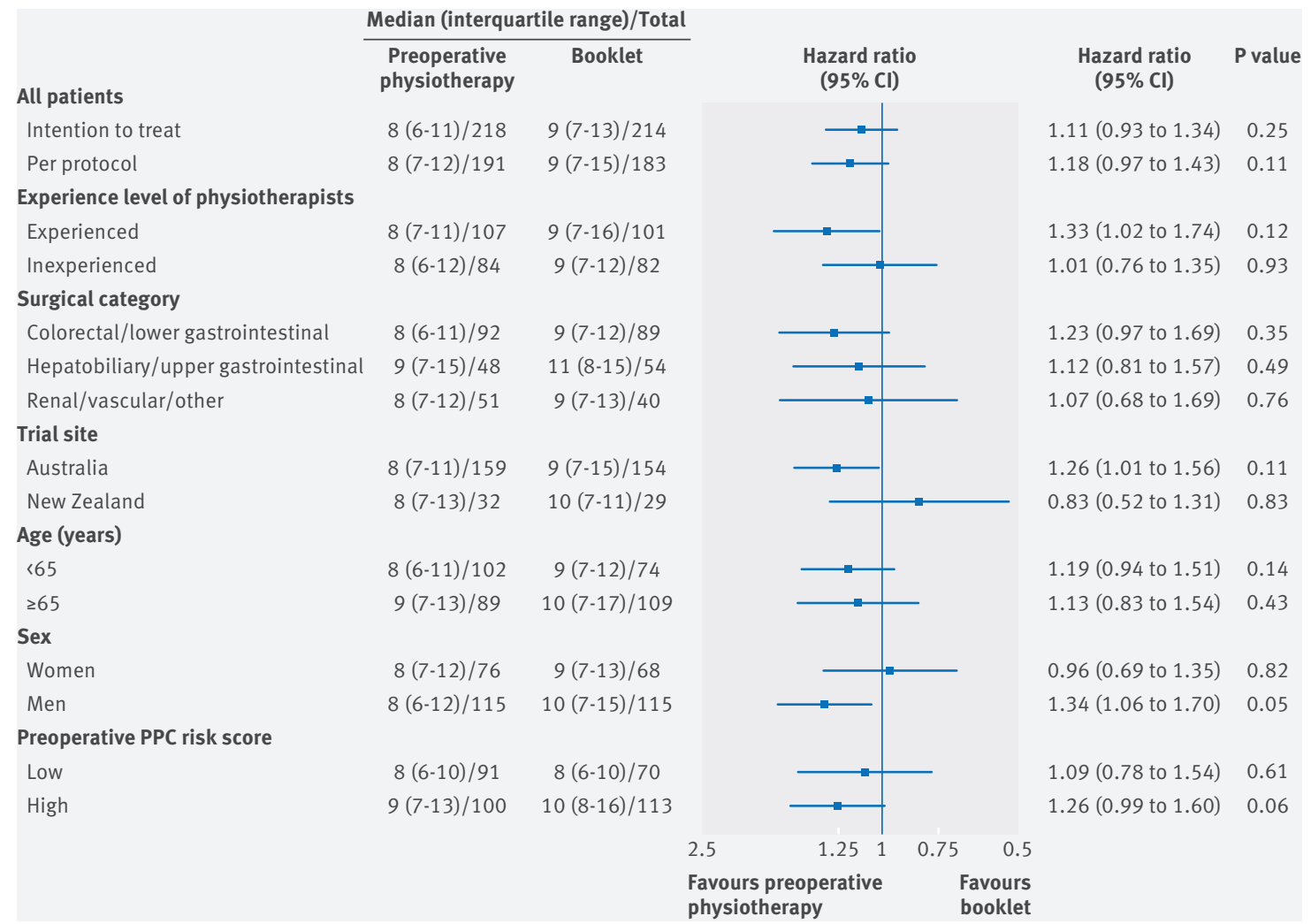

Fig 5 | Sensitivity analysis of subgroup effects on hospital length of stay. Data are adjusted for age, respiratory comorbidity, and upper gastrointestinal surgery. PPC=postoperative pulmonary complication 


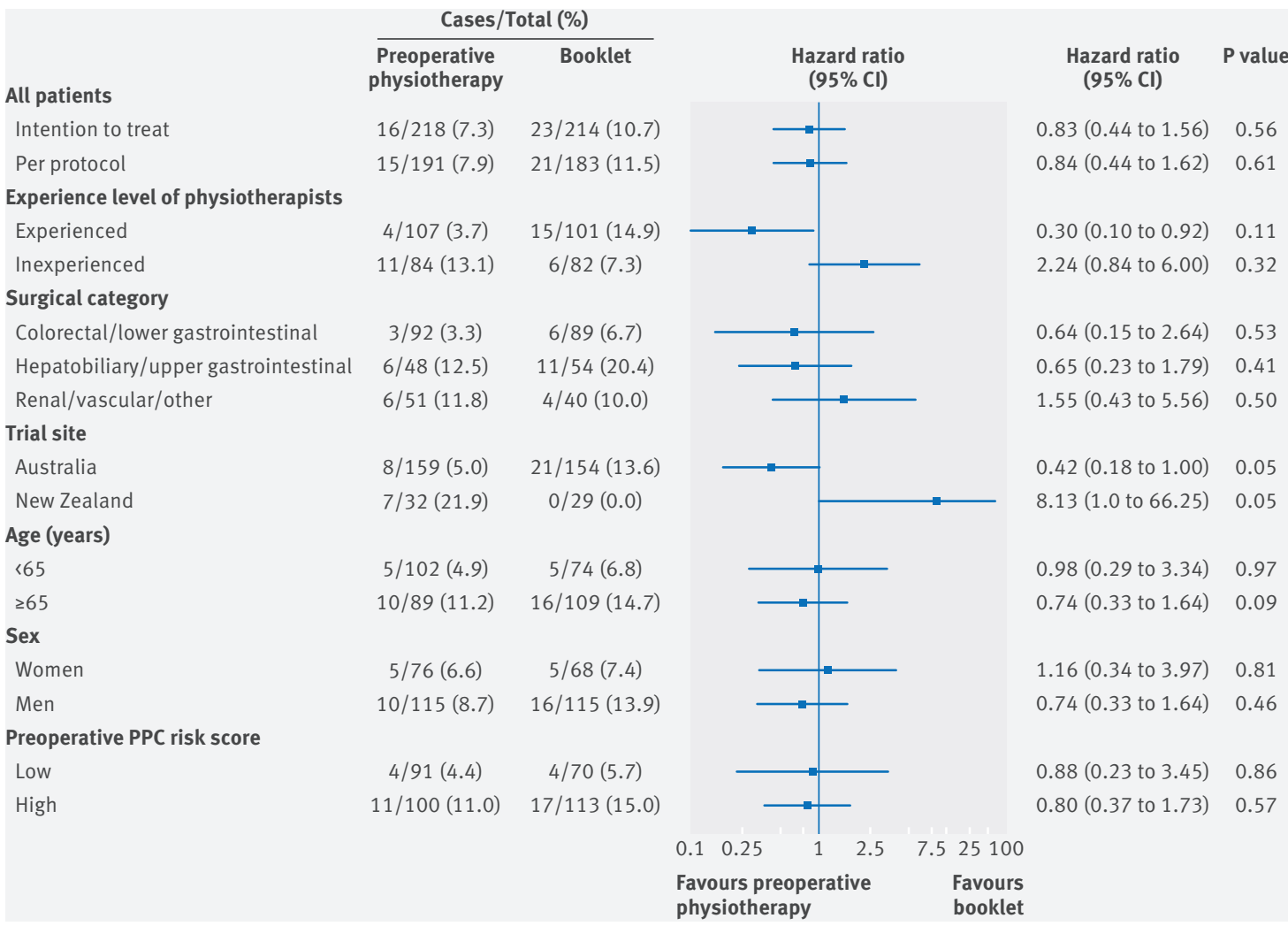

Fig 6 | Sensitivity analysis of subgroup effects on 12 month all cause mortality. Data are adjusted for age, respiratory comorbidity, and upper gastrointestinal surgery. $\mathrm{PPC}=$ postoperative pulmonary complication

and validity of these trials are reduced by the low risk populations, single centre designs, non-masked assessors, and interventions only by experienced practitioners.

Our results are important in the context of considering existing evidence for other methods to prevent PPCs. These include preoperative inspiratory muscle training, "prehabilitation," incentive spirometry, and postoperative chest physiotherapy. Considering how effective preoperative education is in independently reducing PPCs, the benefit attributed to inspiratory muscle training ${ }^{36}$ may come from just educating the patients preoperatively on breathing exercises rather than the effect of the training device itself. Inspiratory muscle training could provide an additive effect to preoperative education, although this currently remains untested. Future research into preventing PPCs will need to standardise the provision of preoperative physiotherapy education to both treatment arms.

\section{Strengths of the trial}

Our trial was specifically designed and powered to address methodological limitations in previous studies.

We included most types of upper gastrointestinal, colorectal, and renal procedures involving traditional full length open incision approaches or via modern minimally invasive methods where smaller length incisions are preferred. From this population, 88\% of eligible patients were entered into the trial, with a 98\% follow-up rate. The three participating sites were representative of the variety of public hospitals in developed countries; a small rural hospital, a medium sized regional tertiary referral hospital, and a large major metropolitan university affiliated hospital. Given this, our cohort is closely representative of the heterogeneous population having upper abdominal surgery. To further promote generalisability of results the intervention was delivered by physiotherapists of varying grades of experience and conducted within an environment reflective of modern perioperative practice where patients attend an outpatient assessment clinic weeks before surgery rather than admission the day before surgery. Assessors, postoperative physiotherapists, and participants were masked to group allocation. To our knowledge we are one of few trials to assess the success of masking (see appendix). We also recorded most known perioperative confounders, including preoperative functional status, intraoperative fluid administered, transfusions, ventilation strategies, and postoperative analgesia and antibiotic management, and we adjusted the results for baseline imbalances in variables known to influence PPCs. To establish efficacy of preoperative education alone, we standardised early mobilisation and successfully removed all postoperative chest physiotherapy modalities.

\section{Implications of findings}

Considering the standardisation of postoperative practice, the most plausible reason for PPC reduction 
in our trial is that the participants performed the breathing exercises as taught preoperatively. This cannot be proved in this study as we opted not to measure postoperative performance of breathing exercises. We considered that measuring such performance could have resulted in a Hawthorne effect by artificially reminding patients to adhere to the prescribed breathing exercises, and results would not be reflective of the pragmatic nature of the intervention. As a proxy measure of compliance, a convenience sample of 29 patients was interviewed on the fifth postoperative day, with $94 \%$ of intervention participants remembering the breathing exercises compared with just $15 \%$ in those who received the

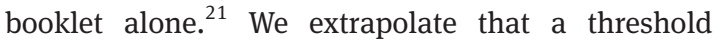
proportion of intervention participants implemented the acquired knowledge provided by the preoperative physiotherapists and performed deep breathing exercises immediately on regaining consciousness from surgery and continued to perform them at a dose necessary to reverse the respiratory pathophysiological changes from surgery, thus preventing PPCs.

Postoperative assisted ambulation in our trial was carefully standardised, as improvements in hospital length of stay are independently attributed to early mobilisation programmes after major surgery. ${ }^{37}$ Early ambulation is also espoused as a possible intervention to prevent PPCs, ${ }^{8}$ although this is not supported at systematic review level. ${ }^{38}$ Our findings, in combination with those of Schaller et al, ${ }^{37}$ suggest specificity of therapy, early ambulation to improve functional recovery, ${ }^{38}$ and respiratory therapy to prevent PPCs. Given the current evidence, postoperative early ambulation cannot be confidently supported as the only method to prevent PPCs; rather, preoperative physiotherapy education should be considered a primary step in PPC prophylaxis for all patients awaiting upper abdominal surgery.

Observational studies associate PPC incidence with increased hospital length of stay. ${ }^{3-10}$ In our study, despite the incidence of PPCs being halved, a statistically significant reduction in length of stay was not detected in the overall population. Possible explanations for this apparent paradox are that previously reported associative data between PPCs and length of stay is unadjusted for other factors that may influence both outcomes, such as surgical category, age, comorbidities, and other concurrent complications. The independent impact of PPCs to affect length of stay may be less than previously reported when accounting for confounding factors. Hospital stay is an outcome with complex multifactorial reasons for determination, and after abdominal surgery the standard deviation is wide. For our population the average length of stay was 11.4 (SD 11.0) days, with a range of 1 to 105 days. To determine a statistically significant difference in length of stay requires a larger sample size or meta-analysis to confirm effect. It may also be that we measured total combined acute and subacute length of stay. Specific subset effects may apply to acute length of stay only.
Despite these limitations, exploratory subgroup analysis of our population revealed that in cohorts with stronger reductions in PPCs attributable to the intervention there was also a corresponding stronger signal to a reduction in length of stay. This suggests that our length of stay findings may be limited by sample size and heterogeneous response rates rather than by a lack of effect from the intervention. Similarly, point estimates across almost all other secondary outcomes in our trial favoured the intervention group, with sensitivity analyses strengthening these relations further. Subgroups with the greatest reduction in PPCs had a consistent signal towards improved secondary outcomes favouring the intervention group. Again this may be an indication that secondary outcome results are limited by sample size rather than by a lack of effect.

Our study has repeated the reported association between PPCs and in-hospital and 30 day mortality, ${ }^{3-5}$ and to our knowledge is the first prospective study to show an association between PPCs in the early postoperative period to 12 month all cause mortality. ${ }^{39}$ Our trial is also the first to find a signal of improved survival attributable to an intervention that reduces the incidence of PPCs, although, considering the low event rates, our study was not adequately powered, nor was it intended to, mortality being an exploratory secondary outcome. The 12 month mortality effect size in our trial was an absolute risk reduction of $5 \%(12 \%$ v 7\%). This would require more than 1000 participants to confirm the effect of preoperative physiotherapy to reduce 12 month mortality. Future studies in prophylactic interventions to prevent PPCs could consider being powered a priori to detect these small, yet arguably clinically important, differences in mortality.

\section{Recommendations for future research}

We recommend that future research is directed towards, firstly, investigating the improved postoperative outcomes dependent on the experience level of physiotherapists providing the preoperative education; for example, is it the way an experienced physiotherapist delivers the intervention, or is it due to repetition and practice of delivering the intervention? The experienced physiotherapist provided the intervention 124 times, compared with a maximum 25 for one of the junior physiotherapists. Many practitioner dependent interventions have a learning curve, including surgery, where surgeon experience is associated with improved morbidity and mortality. ${ }^{40}$ A similar relationship might exist in preoperative education. Preoperative education provided by two physiotherapists, including the most experienced, was found to be highly memorable and impactful for patients. ${ }^{21}$ The treatment integrity of the education and training provided by other physiotherapists in this trial was not checked or graded. Considering the effect gradient according to experience level, further research is required to assess the repeatability of this intervention to ensure that it is provided with a similar 
degree of rigour across all treating therapists. Secondly, preoperative education needs to be validated in other elective surgical populations such as cardiothoracic surgery and neurosurgery.

Considering the strong association between PPCs and mortality and the consistent findings across three trials, four countries, and 1000 patients ${ }^{17} 18$ that preoperative education significantly reduces PPCs; we recommend that future studies should investigate additional PPC prophylactic interventions to augment preoperative physiotherapy education, particularly targeting high risk patients. The PPC rate in high risk patients in our intervention group, although halved, was still 19\%. Considering the high mortality association with PPCs, more urgently needs to be done to prevent PPCs in high risk patients, over and above preoperative physiotherapy education and postoperative ambulation alone.

\section{Limitations of this trial}

Despite concerted methodological efforts to ensure internal validity of the trial, baseline imbalances did exist between the groups. This could just be a chance bias or a failure of true randomisation. Mathematical modelling finds that even with true randomisation, there is a $72.4 \%$ probability of two or more uneven covariates between groups if 50 covariates are included. Therefore, there is a moderate to high likelihood that maldistributions between groups occurred simply by chance. It is also possible, although unlikely, that physiotherapists opened envelopes and deliberately randomised patients prone to PPCs to the control group. ${ }^{41}$ Methods of random allocation less prone to selection bias include telephone or web based systems. We chose to use sealed envelopes as our trial was minimally funded and clinician initiated, and reliable internet access at all sites was not always ensured. Envelopes were considered the most feasible, low tech, and cost effective option to conceal the randomisation order. An independent audit of our randomisation process found no evidence of a failure in sequential allocation (see appendix). Our results were adjusted to control for prespecified confounders imbalanced at baseline; however, our trial could have been further improved by using stratified randomisation according to known confounders-for example, surgical category and respiratory comorbidity. This would have ensured equal distribution at baseline.

Several aspects of our trial also limit generalisability. We excluded non-English speakers and only conducted our trial in developed Western countries. It cannot be extrapolated that preoperative education would be effective with the use of interpreters, in a different social-cultural context, through different modes such as visual recordings or group sessions, or with health professionals other than physiotherapists.

Additionally, despite our trial being multicentred, a large proportion of participants were recruited at a single hospital in Australia. Our trial could have been strengthened with equal distribution of representation from other sites and involvement from other countries.
At the New Zealand site, the reduction in PPCs was less than at Australian sites. It is possible that this was due to the difference in experience level of the preoperative physiotherapists, although the $95 \%$ confidence interval is within the bounds of PPC risk reduction at the other sites, and may rather be a function of a limited sample. The New Zealand site also had established enhance recovery after surgery pathways, ${ }^{19}$ unlike the two Australian sites, which could explain the difference in intravenous fluid amounts, epidural usage, and the lower PPC incidence in the control group (13.8\%). Despite the lower PPC baseline risk, subgroup analysis suggests that across the whole trial sample both high and low risk patients have a similar relative risk reduction of PPCs given preoperative physiotherapy education.

\section{Conclusions and implications for practice}

Our trial provides strong evidence that preoperative education and training delivered within six weeks of open upper abdominal surgery by a physiotherapist reduces the incidence of PPCs, including hospital acquired pneumonia, within the first 14 days after surgery. Our format of preoperative physiotherapy education and training was a single 30 minute intervention with minimal potential to harm and provided within existing multidisciplinary hospital clinics that patients are already required to attend before surgery. These results are directly applicable to the tens of millions of patients listed for elective major abdominal surgery worldwide. This service could be considered for all patients awaiting upper abdominal surgery.

\section{AUTHOR AFFILIATIONS}

${ }^{1}$ Department of Physiotherapy, Launceston General Hospital, Launceston, TAS, 7250, Australia

${ }^{2}$ Department of Physiotherapy, The University of Melbourne, Melbourne, VIC, 3010, Australia

${ }^{3}$ Directorate of Community Integration, Allied Health and Service Planning, Western Health, Melbourne, VIC, Australia

${ }^{4}$ School of Clinical Sciences, Faculty of Health and Environmental Sciences, Auckland University of Technology, Auckland, New Zealand

${ }^{5}$ Physiotherapy Department, North Shore Hospital, Waitemata District Health Board, Auckland, New Zealand

${ }^{6}$ Physiotherapy Department, North West Regional Hospital, Burnie, TAS, Australia

${ }^{7}$ Clifford Craig Foundation, Launceston General Hospital, Launceston, TAS, Australia

${ }^{8}$ School of Health Sciences, University of Tasmania, Launceston, TAS, Australia

${ }^{9}$ Anaesthesia Perioperative and Pain Medicine Unit, Melbourne Medical School, The University of Melbourne, Melbourne, VIC Australia

${ }^{10}$ Melbourne School of Health Sciences, The University of Melbourne, VIC, Australia

${ }^{11}$ Peter MacCallum Cancer Centre, Melbourne, VIC, Australia

We thank the LIPPSMAck POP research assistants, Kate Sullivan and Bronte Biggins-Tosch, for their dedication and thoroughness throughout the trial; the medical students at the University of Tasmania School of Medicine and Leanne Fung (MD)for working as masked assessors; all physiotherapists at the participating sites for assisting in providing the interventions and delivering the standardised postoperative ambulation protocol over the two year trial period; and the participants for their contribution to knowledge in this area. 
Contributors: IB conceived and designed the study, coordinated the trial, prepared the first draft of the manuscript, and was responsible for the final manuscript. IB, LB, EHS, JR, and LD developed the protocol. IB, JR, CH, and LA recruited the patients and acquired the data, and were responsible for protocol adherence and managing the trial at each of the sites. IB and IKR did the statistical analysis. IB, EHS, LB, JR, IKR, DS, and LD analysed and interpreted the data. All authors revised manuscript drafts, approved the final manuscript, and contributed intellectually important content. IB is the guarantor of the paper and takes responsibility for the integrity of the work as a whole, from inception to published article.

Funding: This study was an investigator initiated trial funded by competitive research grants from the Clifford Craig Foundation, Launceston, Australia, the University of Tasmania (virtual Tasmanian Academic Health Science Precinct), Tasmania, Australia, and the Awhina Contestable Research Grant from the Waitemata District Health Board and Three Harbours Health Foundation, Auckland, New Zealand, Support was provided from departmental sources at each participating study centre (Launceston General Hospital, North West Regional Hospital, North Shore Hospital) and through sponsorship by the Tasmanian Health Service-North to support IB for the period of the trial. The funding sources had no controlling role in the study design, data collection, analysis, interpretation, or report writing. All authors had full access to the data on trial completion and had final responsibility for publication submission.

Competing interests: All authors have completed the ICMJE uniform disclosure form at www.icmje.org/coi disclosure.pdf and declare that IB received grants from the Clifford Craig Foundation (CCF), University of Tasmania, and Waitemata District Health Board to fund participating sites for physiotherapists to provide preoperative interventions outside of current standard care and for research assistants to acquire data. JR, LA, and $\mathrm{CH}$ were also supported by these grants to coordinate the project at their respective sites. IKR receives a salary from the CCF to perform statistical analysis and provide study design advice for studies receiving grants from the CCF. IKR also receives information technology and library services from the University of Tasmania. Neither CCF nor the University of Tasmania have managerial authority over IKR's work.

Ethical approval: This study was approved by the Human Research Ethics Committee (Tasmania) Network, Tasmania, Australia (H0011911) and the Health and Disability Ethics Committee, New Zealand (14/NTA/233) and informed written consent was given by all patients.

Data sharing: As prespecified a priori in the LIPPSMAck POP published protocol we welcome independent statistical analysis of our findings and provide open access to our anonymised primary dataset as an appendix. Participants gave informed consent for data sharing with organisations that submit a proposal for post hoc data analysis to the LIPPSMAck POP investigators and receive ethical clearance from their host institution and an approved amendment to the original ethics approvals provided by the two source ethics committees. Following this and on request (ianthe.boden@ths.tas.gov.au), the investigators will share the extended anonymised dataset (with associated coding library). Any published peer reviewed manuscripts derived from post hoc analysis of these shared data must list the LIPPSMAck POP investigators as coauthors.

Transparency: The lead author (IB) affirms that this manuscript is an honest, accurate, and transparent account of the study being reported; that no important aspects of the study have been omitted; and that any discrepancies from the study as planned (and, if relevant, registered) have been explained.

This is an Open Access article distributed in accordance with the Creative Commons Attribution Non Commercial (CC BY-NC 4.0) license, which permits others to distribute, remix, adapt, build upon this work non-commercially, and license their derivative works on different terms, provided the original work is properly cited and the use is noncommercial. See: http://creativecommons.org/licenses/by-nc/4.0/.

1 Weiser TG, Regenbogen SE, Thompson KD, et al. An estimation of the global volume of surgery: a modelling strategy based on available data. Lancet 2008:372:139-44. doi:10.1016/S01406736(08)60878-8

2 Hemmes SN, Gama de Abreu M, Pelosi P, Schultz MJ, PROVE Network Investigators for the Clinical Trial Network of the European Society of Anaesthesiology. High versus low positive end-expiratory pressure during general anaesthesia for open abdominal surgery (PROVHILO trial): a multicentre randomised controlled trial. Lancet 2014:384:495-503. doi:10.1016/S0140-6736(14)60416-5
3 Schultz MJ, Hemmes SN, Neto AS, et al, LAS VEGAS investigators. Epidemiology, practice of ventilation and outcome for patients at increased risk of postoperative pulmonary complications: LAS VEGAS - an observational study in 29 countries. Eur J Anaesthesiol 2017;34:492-507. doi:10.1097/ EJA.00000000000000646

4 Neto AS, Hemmes SN, Barbas CS, et al, PROVE Network investigators. Incidence of mortality and morbidity related to postoperative lung injury in patients who have undergone abdominal or thoracic surgery: a systematic review and meta-analysis. Lancet Respir Med 2014;2:1007-15. doi:10.1016/S2213-2600(14)70228-0

5 Fernandez-Bustamante A, Frendl G, Sprung J, et al. Postoperative Pulmonary Complications, Early Mortality, and Hospital Stay Following Noncardiothoracic Surgery: A Multicenter Study by the Perioperative Research Network Investigators. JAMA Surg 2017:152:157-66. doi:10.1001/jamasurg.2016.4065

6 Fleisher LA, Linde-Zwirble WT. Incidence, outcome, and attributable resource use associated with pulmonary and cardiac complications after major small and large bowel procedures. Perioper Med (Lond) 2014;3:7. doi:10.1186/2047-0525-3-7

7 Scholes RL, Browning L, Sztendur EM, Denehy L. Duration of anaesthesia, type of surgery, respiratory co-morbidity, predicted V02max and smoking predict postoperative pulmonary complications after upper abdominal surgery: an observational study. Aust J Physiother 2009;55:191-8. doi:10.1016/500049514(09)70081-9

8 Haines KJ, Skinner EH, Berney S, Austin Health POST Study Investigators. Association of postoperative pulmonary complications with delayed mobilisation following major abdominal surgery: an observational cohort study. Physiotherapy 2013;99:119-25. doi:10.1016/j.physio.2012.05.013

9 Browning L, Denehy L, Scholes RL. The quantity of early upright mobilisation performed following upper abdominal surgery is low: an observational study. Aust J Physiother 2007:53:47-52 doi:10.1016 S0004-9514(07)70061-2

10 Parry S, Denehy L, Berney S, Browning L, Austin Health PostOperative Surveillance Team (POST) Investigators. Clinical application of the Melbourne risk prediction tool in a high-risk upper abdominal surgical population: an observational cohort study. Physiotherapy 2014;100:47-53. doi:10.1016/i.physio.2013.05.002

11 Mackay MR, Ellis E, Johnston C. Randomised clinical trial of physiotherapy after open abdominal surgery in high risk patients. Aust J Physiother 2005;51:151-9. doi:10.1016/S00049514(05)70021-0

12 Silva YR, Li SK, Rickard MJ. Does the addition of deep breathing exercises to physiotherapy-directed early mobilisation alter patient outcomes following high-risk open upper abdominal surgery? Cluster randomised controlled trial. Physiotherapy 2013;99:187-93. doi:10.1016/j.physio.2012.09.006

13 Miskovic A, Lumb AB. Postoperative pulmonary complications. $\mathrm{Br}$ J Anaesth 2017;118:317-34. doi:10.1093/bja/aex002

14 Pasquina P, Tramèr MR, Granier J-M, Walder B. Respiratory physiotherapy to prevent pulmonary complications after abdominal surgery: a systematic review. Chest 2006;130:1887-99. doi:10.1378/chest.130.6.1887

15 Ball L, Bos LD, Pelosi P. High-flow nasal cannula in the postoperative period: is positive pressure the phantom of the OPERA trial? Intensive Care Med 2017;43:119-21. doi:10.1007/s00134016-4627-6

16 Patman S, Bartley A, Ferraz A, Bunting C. Physiotherapy in upper abdominal surgery - what is the current practice in Australia? Arch Physiotherapy, 2017:7-11. https://doi.org/10.1186/s40945-0170039 -

17 Fagevik Olsén M, Hahn I, Nordgren S, Lönroth H, Lundholm K. Randomized controlled trial of prophylactic chest physiotherapy in major abdominal surgery. Br J Surg 1997;84:1535-8. doi:10.1002/ bjs.1800841111

18 Samnani SS, Umer MF, Mehdi SH, Farid FN. Impact of Preoperative Counselling on Early Postoperative Mobilization and Its Role in Smooth Recovery. Int Sch Res Notices 2014;2014:250536. doi:10.1155/2014/250536

19 Gustafsson UO, Scott MJ, Schwenk W, et al, Enhanced Recovery After Surgery (ERAS) Society, for Perioperative Care, European Society for Clinical Nutrition and Metabolism (ESPEN), International Association for Surgical Metabolism and Nutrition (IASMEN). Guidelines for perioperative care in elective colonic surgery: Enhanced Recovery After Surgery (ERAS( (囚)) Society recommendations. World J Surg 2013;37:259-84. doi:10.1007 s00268-012-1772-0

20 Boden I, Browning L, Skinner EH, et al. The LIPPSMAck POP (Lung Infection Prevention Post Surgery-Major Abdominal-with PreOperative Physiotherapy) trial: study protocol for a multi-centre randomised controlled trial. Trials 2015;16:1-15. doi:10.1186/ s13063-015-1090-6 
21 Boden I, El-Ansary D, Zalucki N, et al. Physiotherapy education and training prior to upper abdominal surgery is memorable and has high treatment fidelity: a nested mixed-methods randomised-controlled study. Physiotherapy 2017; published online 30 Aug. https://doi. org/10.1016/j.physio.2017.08.008

22 Brooks D, Parsons J, Newton J, et al. Discharge criteria from perioperative physical therapy. Chest 2002;121:488-94 doi:10.1378/chest.121.2.488

23 Sopena N Sabrià M. Neunos 2000 Study Group. Multicenter study of hospital-acquired pneumonia in non-ICU patients. Chest 2005;127:213-9. doi:10.1378/chest.127.1.213

24 Fiore JFIr, Faragher IG, Bialocerkowski A, Browning L, Denehy L. Time to readiness for discharge is a valid and reliable measure of shortterm recovery after colorectal surgery. World I Surg 2013;37: 2927-34. doi:10.1007/s00268-013-2208-1

25 McHorney CA, Ware JEJr, Lu JF, Sherbourne CD. The MOS 36-item Short-Form Health Survey (SF-36): III. Tests of data quality, scaling assumptions, and reliability across diverse patient groups. Med Care 1994:32:40-66. doi:10.1097/00005650-199401000-00004

26 Rankin SL, Briffa TG, Morton AR, Hung J. A specific activity questionnaire to measure the functional capacity of cardiac patients. Am J Cardiol 1996:77:1220-3. doi:10.1016/S0002-9149(97)89157-6

27 Hughes M, Coolsen MM, Aahlin EK, et al. Attitudes of patients and care providers to enhanced recovery after surgery programs after major abdominal surgery. I Surg Res 2015;193:102-10. doi:10.1016/i.jss.2014.06.032

28 Stern C, Lockwood C. Knowledge retention from preoperative patient information. Int J Evid Based Healthc 2005;3:45-63.

29 Hall M, Migay A-M, Persad T, et al. Individuals' experience of living with osteoarthritis of the knee and perceptions of total knee arthroplasty. Physiother Theory Pract 2008;24:167-81. doi:10.1080/09593980701588326

30 Keulers BJ, Scheltinga MR, Houterman S, Van Der Wilt GJ, Spauwen PH. Surgeons underestimate their patients' desire for preoperative information. World I Surg 2008;32:964-70. doi:10.1007/s00268-008-9581-1

31 Grabitz SD, Farhan HN, Ruscic KJ, et al. Dose-dependent protective effect of inhalational anesthetics against postoperative respiratory complications: a prospective analysis of data on file from three hospitals in New England. Crit Care Med 2017;45:e30-9. doi:10.1097/CCM.0000000000002015

32 Neto AS, Hemmes SN, Barbas CS, et al, PROVE Network Investigators. Association between driving pressure and development of postoperative pulmonary complications in patients undergoing mechanical ventilation for general anaesthesia: a meta-analysis of individual patient data. Lancet Respir Med 2016:4:272-80. doi:10.1016/S2213-2600(16)00057-6
33 Condie E, Hack K, Ross A. An investigation of the value of routine provision of postoperative chest physiotherapy in non-smoking patients undergoing elective abdominal surgery. Physiotherapy 1993;79:547-52doi:10.1016/S00319406(10)60295-7.

34 Mans CM, Reeve JC, Elkins MR. Postoperative outcomes following preoperative inspiratory muscle training in patients undergoing cardiothoracic or upper abdominal surgery: a systematic review and meta analysis. Clin Rehabil 2015;29:426-38. doi:10.1177/0269215514545350

35 Castillo R, Haas A. Chest physical therapy: comparative efficacy of preoperative and postoperative in the elderly. Arch Phys Med Rehabil 1985:66:376-9.

36 Katsura M, Kuriyama A, Takeshima T, Fukuhara S, Furukawa TA. Preoperative inspiratory muscle training for postoperative pulmonary complications in adults undergoing cardiac and major abdominal surgery. Cochrane Database Syst Rev 2015;(10):CD010356

37 Schaller S), Anstey M, Blobner M, et al, International Early SOMSguided Mobilization Research Initiative. Early, goal-directed mobilisation in the surgical intensive care unit: a randomised controlled trial. Lancet 2016;388:1377-88. doi:10.1016/ S0140-6736(16)31637-3

38 Castelino T, Fiore JF Jr, Niculiseanu P, Landry T, Augustin B, Feldman LS. The effect of early mobilization protocols on postoperative outcomes following abdominal and thoracic surgery: A systematic review. Surgery 2016;159:991-1003. doi:10.1016/ j.surg.2015.11.029

39 Khuri SF, Henderson WG, DePalma RG, Mosca C, Healey NA, Kumbhani DJ. Participants in the VA National Surgical Quality Improvement Program. Determinants of long-term survival after major surgery and the adverse effect of postoperative complications. Ann Surg 2005:242:326-41, discussion 341-3.

40 Schmidt CM, Turrini O, Parikh P, et al. Effect of hospital volume, surgeon experience, and surgeon volume on patient outcomes after pancreaticoduodenectomy: a single-institution experience. Arch Surg 2010;145:634-40. doi:10.1001/ archsurg.2010.118

41 Clark L, Fairhurst C, Torgerson DJ. Allocation concealmen in randomised controlled trials: are we getting better? BMJ 2016;355:i5663. doi:10.1136/bmj.i5663

Supplementary appendix: additional information 